\title{
Taxonomias de Geometria da Arquitetura Contemporânea como elementos didáticos para a prática do Projeto Paramétrico
}

\section{Taxonomies of the Contemporary Architecture Geometry as training aids for the practice of Parametric Design}

\author{
Janice de Freitas Pires \\ Universidade Federal de Pelotas, Brasil \\ janicefpires@hotmail.com
}

\author{
Alexandre Gonçalves \\ Universidade Federal de Santa Catarina, Brasil \\ alexandre.I.gonçalves@gmail.com
}

\author{
Alice Therezinha Cybis Pereira \\ Universidade Federal de Santa Catarina, Brasil \\ acybis@gmail.com
}

\begin{abstract}
This work has a didactic approach in teaching digital graphic representation for Architectural Design. Seeks to identify structures to know that support geometric concepts employed in works of contemporary architecture. Faced with technological advances and the recent insertion of parametric design in offices and schools of architecture, the study also aims to support the recognition techniques parametric modeling related to such concepts. How explicit methodology to know structures are adopted, taxonomies and ontologies, we consider the potential of these structures to explain the treaty knowledge and facilitate the association of educational materials on the topic in online systems, supporting learning processes of the agents involved (students and teachers).
\end{abstract}

Keywords: Architecture Education; Parametric Modeling; Contemporary Architecture; Taxonomy; Ontology.

\section{Introdução}

Nos últimos anos o advento de novas técnicas de representação gráfica digital e de simulação, proporcionado pelo desenvolvimento das tecnologias digitais, tem mudado rapidamente o modo de produção da arquitetura. Esta mudança abrange o processo de projeto em arquitetura desde a fase de concepção, passando por estudos de comportamento e resistência estrutural do objeto concebido, até a materialização do edifício, através de protótipos de estudo ou para a construção propriamente dita de elementos componentes da obra.

Segundo Oliveira e Pereira (2011) a amplitude de técnicas digitais disponíveis na atualidade, seja para a representação gráfica, simulação ou manufatura, traz possibilidades concretas de aplicação no ensino do processo de projeto de Arquitetura, por permitir que o projetista (estudante) explore claramente e com maior precisão os aspectos que o envolvem.

Sistemas como o BIM (Building Information Modelling) permitem manipular, simultaneamente e em conjunto, as informações e representações definidas em cada uma das etapas do projeto; Sistemas paramétricos e de geração de formas, permitem ter um maior controle das possibilidades de geração de geometrias complexas e um maior número de alternativas de projeto para avaliação e seleção do projetista; Sistemas de prototipagem rápida e fabricação digital possibilitam analisar conceitualmente propostas de projeto, avaliar e assim materializar a proposta em termos construtivos; Sistemas de simulação de desempenho ou performance da obra, tais como os de energia, fluxo de vento, de estrutura, são alguns exemplos de tecnologias de apoio ao processo de projeto em arquitetura. As iniciativas para a inserção de tais tecnologias no contexto de ensino do projeto de arquitetura tem se concretizado pelos grupos de pesquisa das referidas escolas, muitas vezes através de parcerias que buscam trazer profissionais liberais e/ou pesquisadores com experiência e conhecimentos específicos de aplicação.

Por outro lado, ainda é recorrente nas escolas de arquitetura no Brasil a tradição do ensino de representação gráfica com enfoque exclusivo em geometria descritiva, e seus métodos projetivos sobre um ou dois planos. Para Pottmann et al (2007), isso que acaba por limitar os aspectos de exploração da forma e de geração de geometrias complexas.

Em termos de currículo de arquitetura, poucas são as iniciativas que buscam integrar diferentes técnicas de representação gráfica para o estudo do objeto arquitetônico, principalmente em relação aos aspectos geométricos como base de conhecimento para o projeto paramétrico. Considera-se a necessidade de um avanço nos métodos de ensino buscando abarcar principalmente o aspecto de configuração da forma (Pires e Borda, 2009; Pires, 2010; Pottmann et al, 2007).

Em tais processos de ensino e aprendizagem, há dificuldade de encontrar materiais didáticos estruturados que explicitem este conhecimento, de maneira a facilitar a sua apropriação e utilização durante o processo de projeto. 
SIGraDi 2016, XX Congress of the Iberoamerican Society of Digital Graphics

9-11, November, 2016 - Buenos Aires, Argentina

Mais recentemente identificou-se também a necessidade de atualizar os métodos de representação, frente às atuais necessidades profissionais, as quais cada vez mais vêm sofrendo influência do avanço tecnológico. Nesse sentido, o projeto paramétrico (Woodbury, 2010) tem se constituído como uma prática nos escritórios de arquitetura e como método de ensino de projeto em contextos educativos. Esta abordagem envolve o conhecimento específico de técnicas de modelagem paramétrica (Monedero, 2000).

Entende-se que, com interesse didático, seja necessária, também, a sistematização deste conhecimento através da representação de obras da arquitetura contemporânea e as respectivas ações projetuais empregadas pelos seus arquitetos.

No contexto que se insere este estudo A Teoria Antropológica da Didática (Chevallard, 1999), através de sua abordagem de estruturas de saber, tem promovido apoio a estruturação de processos de ensino e aprendizagem.

Em trabalhos anteriores (Pires e Borda, 2010; Borda et al, 2010) a explicitação de tais estruturas de saber referentes a representação gráfica digital para o projeto de arquitetura foi associada com a estruturação de taxonomias, as quais, segundo referido por Novo (2007), são estruturas classificatórias do saber, que tem por finalidade permitir a agregação de informações e dados, e o entendimento de como o domínio do conhecimento é organizado em uma área ou saber específico.

A hipótese do estudo é que através de taxonomias e ontologias que tratem da estrutura de conhecimento associada à geometria da arquitetura contemporânea e a modelagem paramétrica, seja possível sistematizar elementos didáticos de apoio ao desenvolvimento do projeto paramétrico.

\section{Referencial Teórico e Metodológico}

Conforme já referido, a partir dos avanços tecnológicos dos últimos anos, novos conceitos da geometria têm-se mostrado potencialmente presentes na arquitetura contemporânea. Estes, segundo Pottmann (2010), apontam para um elevado nível de complexidade geométrica.

Para o autor tais saberes não são abordados e ensinados em cursos de desenho ou Geometria Descritiva nas escolas de arquitetura. Destaca que a recente inserção do Parametric Design (Projeto Paramétrico) exige mais ainda aprofundar na geometria da forma. Sistemas paramétricos e de geração de formas permitem ter um maior controle das possibilidades de geração de geometrias complexas e um maior número de alternativas de projeto para avaliação e seleção do projetista.
Segundo Monedero (2000), o projeto paramétrico é entendido como um processo em que a descrição de um problema é criada usando variáveis. Ao alterar essas variáveis uma gama de soluções alternativas pode ser criada, em seguida, com base em alguns critérios selecionados de uma solução final. Nesta base, pode-se dizer todo o projeto é paramétrico. O autor destaca que, em termos computacionais, é o processo de desenvolvimento de um modelo de computador ou a descrição de um problema de design. Esta representação é baseada em relações entre os objetos controlados por variáveis. Fazer alterações às variáveis resulta em modelos alternativos. A seleção de uma solução é feita com base em alguns critérios que podem ser relacionados com o desempenho, facilidade de construção, requisitos de orçamento, as necessidades do usuário, estética ou uma combinação destes.

De acordo Woodbury (2010), o processo de criação de relacionamentos (necessariamente) requer uma notação formal e introduz conceitos adicionais que não tenham sido previamente considerados como parte do "pensamento de design". O autor considera que Isso pode alargar o âmbito intelectual do projeto.

Em Mitchell (1991) já havia sido enfatizada a necessidade de descrição dos edifícios pelos arquitetos e projetistas para uma avaliação crítica da produção de arquitetura. Vaz (2011) destaca que, de acordo com o pensamento de Mitchell (1991), a partir de uma conceitualização adequada, é possível estabelecer as bases para a descrição de um edifício. Esta descrição irá conter relações e propriedades de partes do edifício, sendo possível se referir a elas diretamente ou indiretamente. Quando essa descrição é feita por meio de sentenças escritas, a conceitualização permanece implícita no vocabulário e na construção das sentenças utilizadas. Para Vaz (2011) outra possibilidade de representação da descrição é por meio de sentenças de lógica de primeira ordem. Neste caso, a conceitualização é explicitada por meio da definição de constantes, variáveis, funções e as relações que se pretende utilizar. $O$ mesmo autor ainda destaca que uma terceira forma de explicitar uma conceitualização seria pela descrição no formato de uma ontologia. Ontologia tem sua origem na Filosofia e foi definida para estudar "o ser" ou "a existência" e suas características básicas; busca quais entidades e que tipos de entidades existem.

Uma ontologia define um vocabulário comum para domínios em que exista a necessidade de compartilhamento de informações; No contexto da Computação, inclui definições interpretáveis por máquinas de conceitos básicos em um domínio e relações entre estes, sendo definida como "Uma especificação formal e explícita de uma conceitualização compartilhada" (Studer et al, 1998).

Uma especificação formal se refere a ser processável por máquina. Explícita refere-se a uma definição sem ambiguidade de todos os conceitos, atributos e relacionamentos. Conceitualização refere-se ao modelo 
conceitual de um dado domínio. E compartilhada se refere a um entendimento compartilhado.

Para definirmos uma ontologia é necessário descrever: classes (ou "coisas") nos vários domínios de interesse; relacionamentos entre estas "coisas"; propriedades (ou atributos) que estas "coisas" devem possuir. A definição de classes e sua hierarquia constituem a taxonomia de uma ontologia.

Ontologias podem ser usadas para analisar determinado conhecimento de domínio. Conforme referido anteriormente, tem se adotado a "Teoria Antropológica da Didática", particularmente a visão estruturada do saber, para analisar saberes no contexto de ensino e aprendizagem da representação gráfica digital para arquitetura. Ao considerar que uma estrutura de saber se constitui por quatro elementos - "problema", "técnicas" de resolução deste problema, "tecnologias" (discursos que produzem e explicam as técnicas) e "teorias" (que produzem e justificam as tecnologias) - esta visão promove o estudo do processo dinâmico de constituição de tal estrutura, associando-se a um único problema, elementos advindos de diversas abordagens.

A Fig. 1 ilustra a constituição de um material didático que aborda um determinado processo de modelagem paramétrica para aplicação em arquitetura, explicitando a estrutura de saber necessária ao seu entendimento.
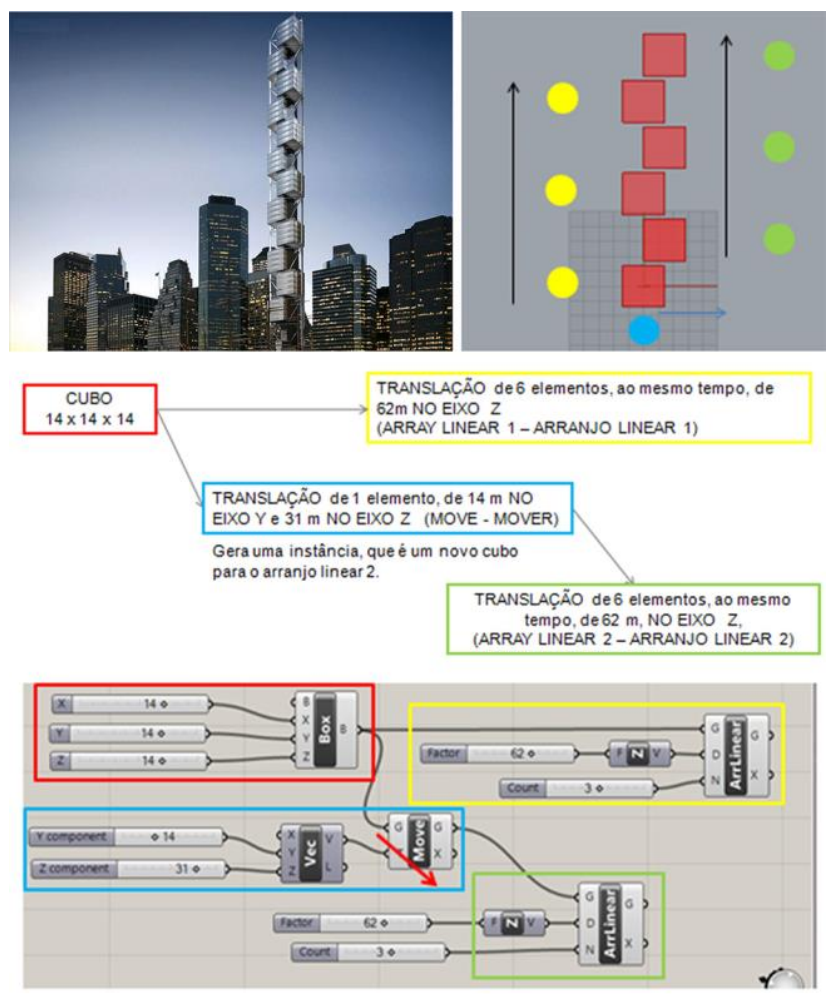

Fig. 1: Material didático que sistematiza processo de modelagem paramétrica de uma obra de Santiago Calatrava. Fonte: Autora, 2015

O material está constituído: por um exemplo de obra de arquitetura e o conceito geométrico associado a tal obra; pela esquematização visual de seu processo de geração; pela organização em formato de mapa conceitual de um algoritmo que descreve as etapas de geração da geometria da obra e o qual se corresponde com a esquematização visual descrita anteriormente; pelo reconhecimento dos elementos tecnológicos de definição paramétrica para este processo de geração; e, por fim, pela definição das relações entre estes elementos e os parâmetros a serem atribuídos. Todo este processo está dividido em grandes etapas que contemplam etapas menores.

O método exemplificado na Fig. 1 ilustra uma aplicação da proposta descrita em Pires e Borda (2010), relativa a estruturação de objetos de aprendizagem. Esta reitera que a identificação das estruturas de saber exige buscar a essência do saber tratado a partir das interconexões entre os conceitos, criando-se uma estrutura que pode ser organizada em palavras-chaves que os representam de modo a delimitarem interconexões mais amplas, o que promoveria diferentes trajetórias de aprendizagem.

A partir destes referenciais teóricos e metodológicos, desenvolve-se uma análise sobre um conhecimento específico de geometria aplicada na arquitetura contemporânea (Burry \& Burry, 2010), utilizando-se modelos de desenvolvimento de ontologias. Estes são dos tipos top-down (definição dos conceitos mais gerais e sua especialização), bottom-up (definição dos conceitos mais específicos e posterior organização em classes mais gerais) e por combinação, os quais definem os conceitos mais relevantes primeiro e então os generaliza e especializa simultaneamente, de maneira apropriada (Studer et al, 1998).

Neste trabalho foi predominantemente utilizado o último tipo. Após a identificação e categorização, as estruturas de saber encontradas ou palavras-chave destacadas foram organizadas por meio de mapas conceituais, com o objetivo de sistematizá-los aplicando uma categorização para o conhecimento que foi analisado. A esta categorização foram adicionados exemplos de obras de arquitetura que se utilizam dos conceitos identificados, conforme descrito por Burry \& Burry (2010). A taxonomia foi então formalizada no software Protegè (http://protege.stanford.edu/) para ser disposta em ambientes na Internet, por meio da Web Ontology Language (OWL), que é utilizada para definição de ontologias para a Web e endossada pelo W3C Web Ontology Working Group (Studer et al, 1998).

\section{Desenvolvimento}

Inicialmente, foi selecionado um conteúdo que exemplifica conceitos geométricos e matemáticos com desenvolvimento recente associado a aplicações arquitetônicas contemporâneas, identificados por Burry \& Burry (2010). Estes, segundo os mesmos autores, se referem ao uso de 'tilings' e 'packings', definidos como "partições do plano ou de um espaço tridimensional" que podem abrigar espaços arquitetônicos. Sobre este conteúdo destacaram-se as palavras-chave que descrevem tais conceitos arquitetônicos e matemáticos. A Fig. 2 ilustra estas aplicações na arquitetura contemporânea. 


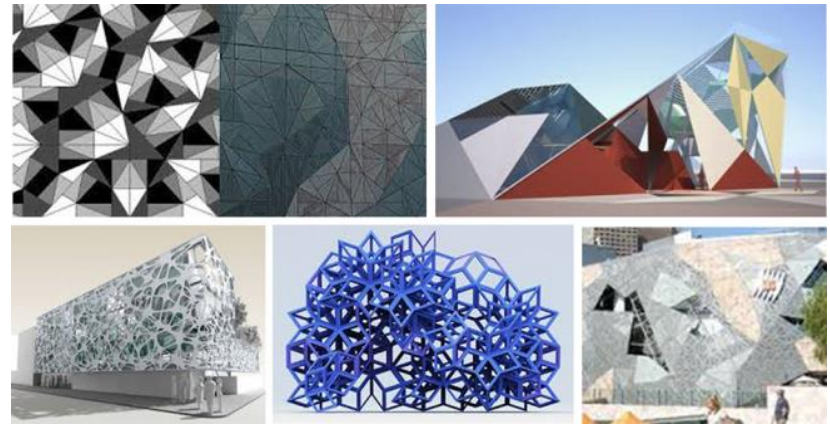

Fig. 2: Obras de arquitetura contemporânea que utilizam conceitos matemáticos. Fonte: Burry \& Burry (2010).

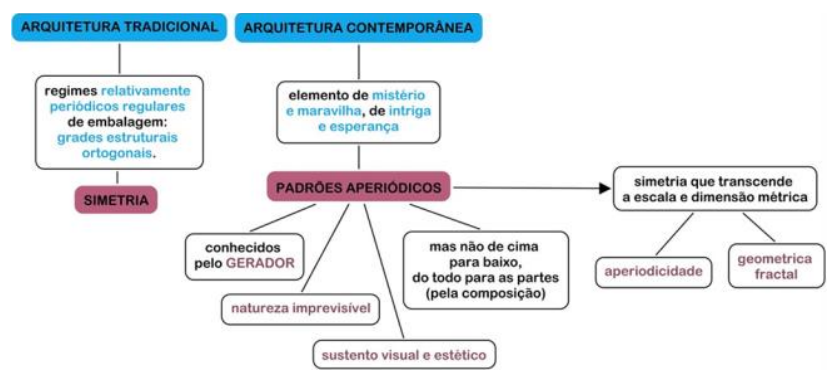

Fig. 3: Mapa conceitual que destaca categorias de palavraschaves associadas a conceitos arquitetônicos (em azul) e geométricos (em bordô) relacionados a aplicação de tilings na arquitetura contemporânea, a partir de Burry \& Burry (2010).

Para destacá-las, aplicou-se cor, de modo a diferenciá-las por categorias, contemplando desde categorias mais gerais até as mais específicas e também a classe de conceitos arquitetônicos (na cor azul, no mapa da Fig. 3), geométricos (na cor bordô, nos mapas das Fig. 3 e 4) e matemáticos (em cor laranja e marrom, no mapa da Fig. 5). Conforme referido anteriormente a classificação por hierarquia buscou identificar tanto a generalidade quanto a especificidade de cada termo, alocando-os nas classes e subclasses identificadas. Os mapas foram construídos utilizando-se a ferramenta CMAP tools (http://cmap.inmc.us/).

O mapa da Fig. 3 foi construído de modo a categorizar os termos identificados no referencial teórico adotado, de maneira a associar a linguagem arquitetônica com a linguagem da geometria. Neste mapa identificam-se as classes Arquitetura Tradicional e Arquitetura Contemporânea, assim como as subclasses Simetria e Padrões Aperiódicos, associados com as classes citadas. Também foram sinalizados em cor outros termos que se referem aos atributos das classes identificadas.

No mapa da Fig. 4 para categorizar dois conceitos geométricos específicos, Packing e Tiling, utilizou-se o modelo bottom-up para o desenvolvimento de ontologias, alocando-se tais conceitos na classe mais geral denominada de Subdivisãodo Espaço. Na sequência foi sendo detalhado cada um destes conceitos, em um modelo top-down, criando-se novas subclasses de termos associadas, tais como Enchimento do espaço e Partição do plano, Espaços habitáveis e Space-frame estrutural.

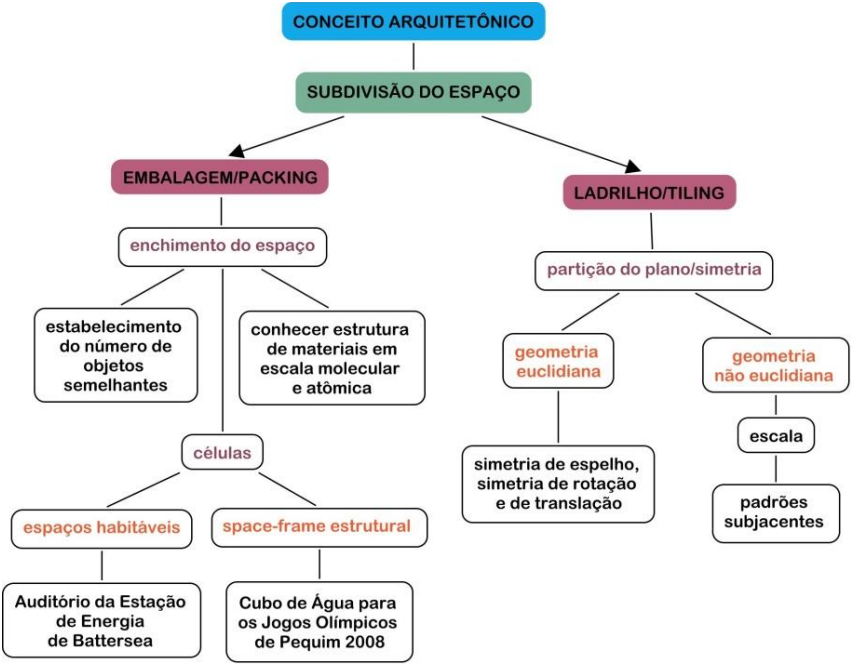

Fig. 4: Mapa conceitual que destaca subcategorias de palavraschaves associadas a conceitos arquitetônicos na arquitetura contemporânea, desenvolvido pelos autores (2015).

O mapa da Fig. 5 busca detalhar o conceito de Tiling, associando as categorias Geometria Euclidiana e Geometria não Euclidiana, e as subcategorias Periódicos e Não periódicos, além dos tipos identificados para cada uma destas subcategorias.

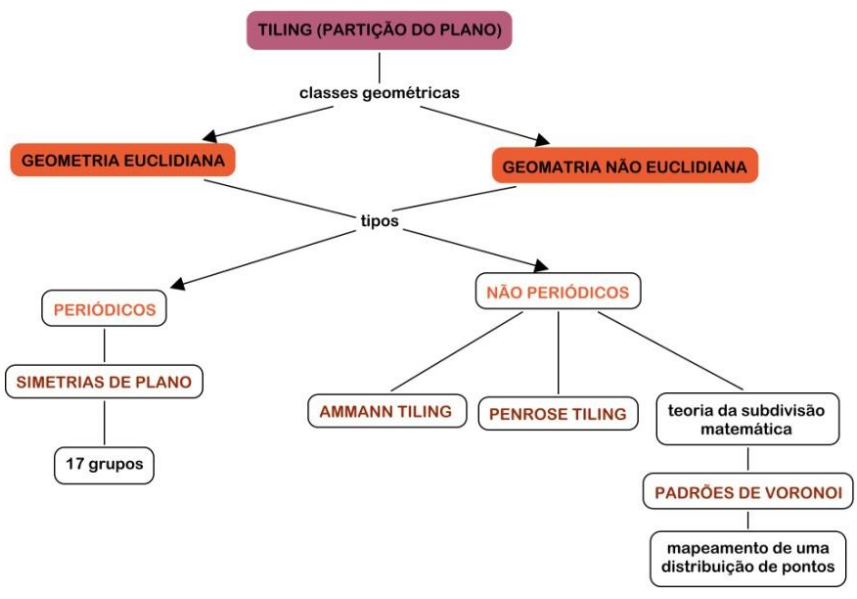

Fig. 5: Mapa conceitual que destaca subcategorias de palavraschaves associadas a conceitos geométricos e matemáticos (em bordô e laranja), relacionados a aplicação de tilings na arquitetura contemporânea, desenvolvido pelos autores (2015).

Após esta identificação e categorização, as estruturas de saber encontradas ou palavras-chave destacadas e organizadas através dos mapas conceituais ilustrados foram reunidas em um único esquema, com o objetivo de sistematizá-los aplicando uma categorização para o conhecimento que foi analisado (Fig. 6). Para isso utilizouse o modelo combinado da abordagem top-down e bottonup, como referido anteriormente. Tal esquema constitui-se em uma taxonomia do saber tratado. A esta taxonomia foram adicionados exemplos de obras de arquitetura que se utilizam dos conceitos identificados, conforme descrito por Burry \& Burry (2010). 


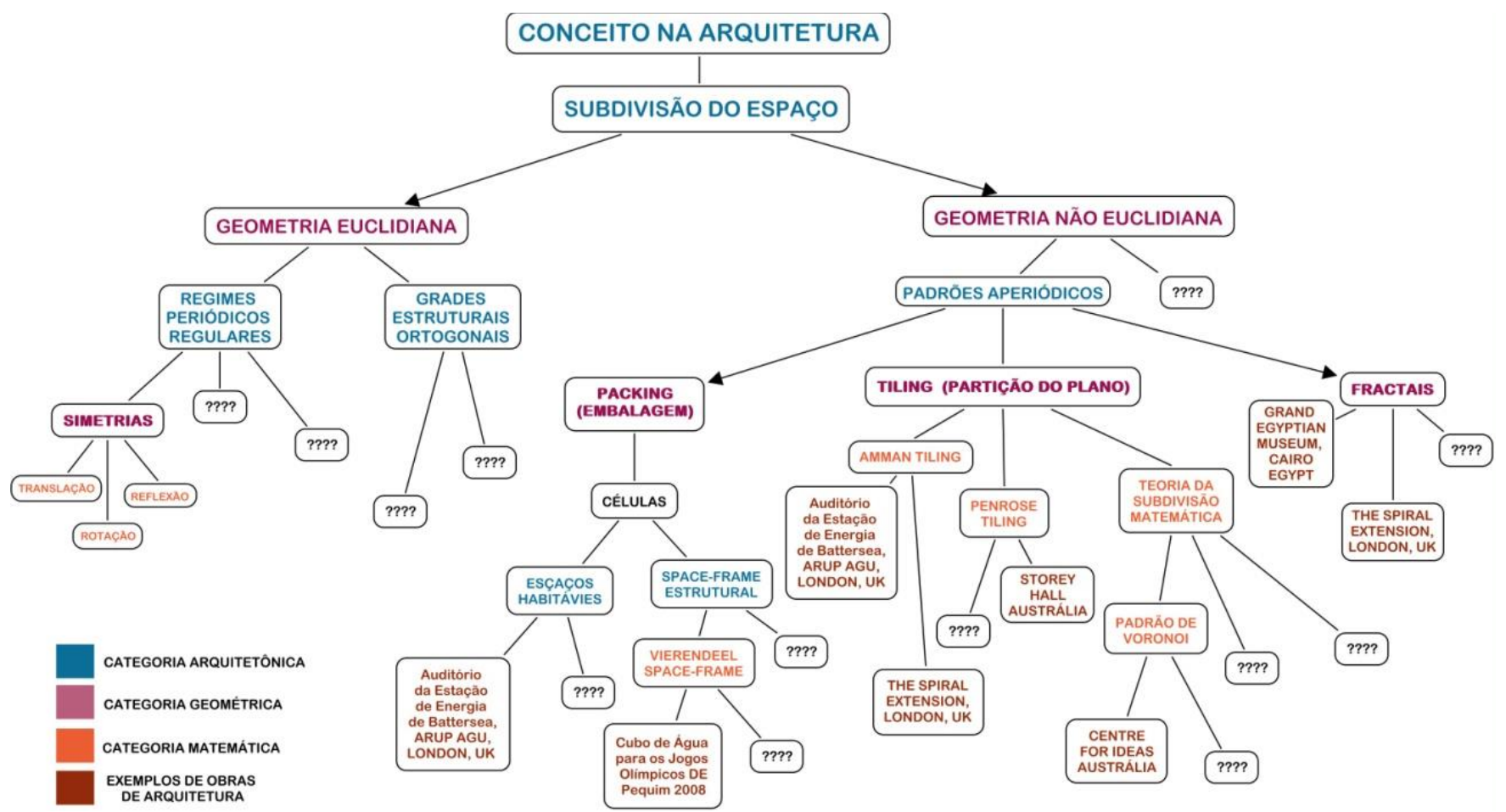

Figura 6: Mapa conceitual que categoriza e classifica em uma taxonomia os conceitos identificados durante o processo de análise, desenvolvido pelos autores, 2015.

Esta taxonomia tanto pode apoiar processos de ensino e aprendizagem como pode dar subsídios a disponibilização de objetos de aprendizagem em ambientes na Internet, desde que a categorização definida e relacionamentos entre os termos permite associar materiais educacionais que contenham os mesmos termos.
Com o objetivo de dispor de uma taxonomia formalizada para ambientes na Internet, transpôs-se a taxonomia para a Web Ontology Language (OWL), linguagem utilizada para definição de ontologias para a Web e endossada pelo W3C Web Ontology Working Group, por meio do software Protegè (fig. 7 e 8). A taxonomia implementada buscou abarcar a estrutura apresentada no mapa da figura 6 .

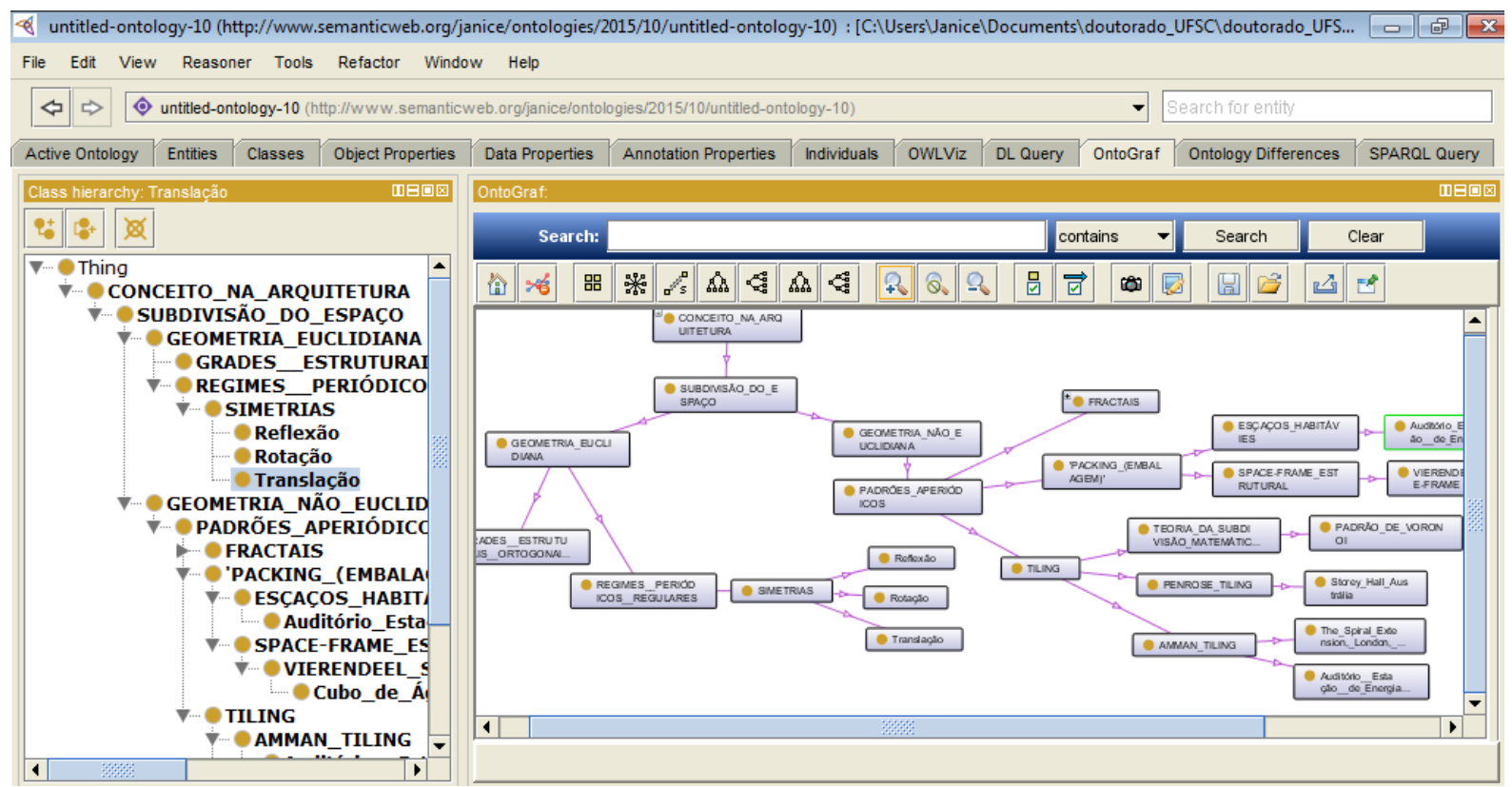

Figura 7: Formalização da taxonomia para web semântica, formato RDF/XML, no software Protegè (http://protege.stanford.edu/), desenvolvido pelos autores (2015). 


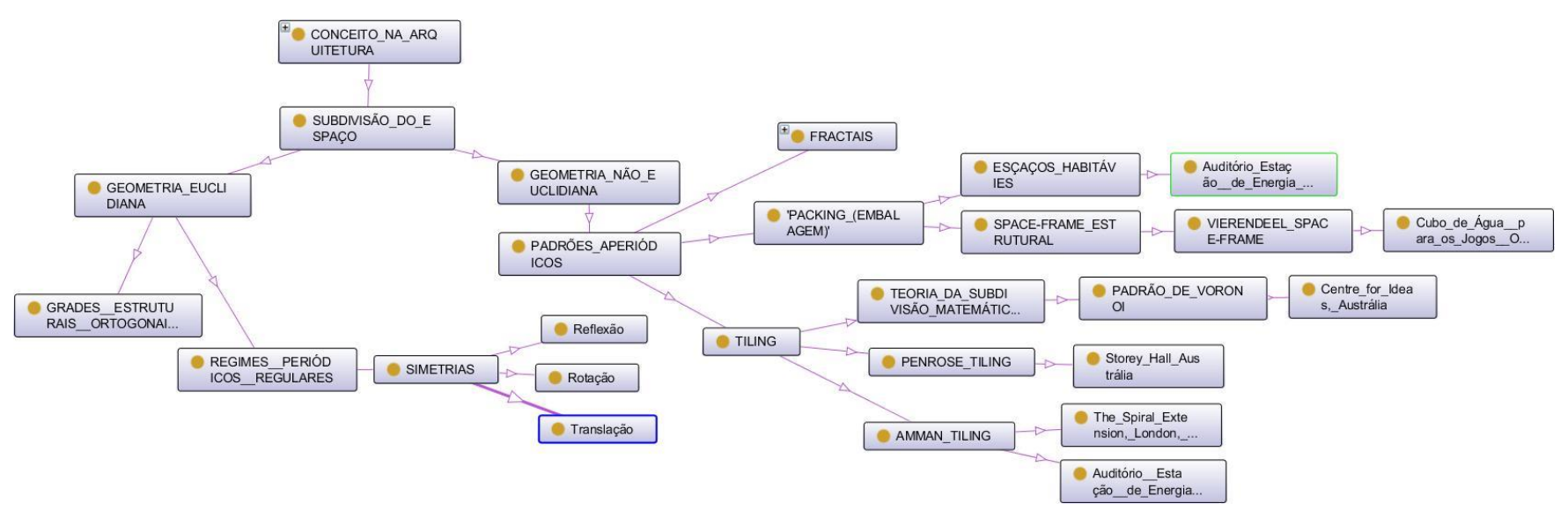

Figura 8: Esquema visual da taxonomia gerado pelo OntoGraf, no software Protegê, desenvolvido pelos autores (2015).

\section{Discussão}

A análise sobre uma estrutura de conhecimento específica de aplicação de novos conceitos matemáticos na arquitetura contemporânea possibilitou identificar: a presença de uma sobreposição das linguagens arquitetônica, geométrica e matemática; a falta de clareza para identificar os tipos envolvidos no saber analisado indicando a necessidade de explicitar saberes que permitam a categorização do conteúdo por tipos, sendo que, a partir do conhecimento abordado em tal material analisado, foi percebida maior facilidade em organizar a terminologia hierarquicamente; e 0 detalhamento insuficiente de determinados termos e conceitos empregados.

O ensaio proposto permitiu compreender que para 0 contexto didático somente a categorização hierárquica não é suficiente para sistematizar a estrutura de saber tratada. Considera-se necessário categorizar por tipos e principalmente explicitar os relacionamentos entre os termos e suas características. Isso vai de encontro a definição de atributos para cada entidade classificada, configurando a necessidade de especificação de uma ontologia.

Conclui-se por fim a pertinência ou necessidade de explicitar em um nível mais profundo a taxonomia desenvolvida, avançando para a descrição de uma ontologia que suporta o conhecimento tratado no material analisado.

Por outro lado, mesmo que a taxonomia constituída não possua a mesma potencialidade de uma ontologia, esta formalização taxonômica em sistemas online de aprendizagem, por explicitar uma terminologia própria, subsidiará tanto a indexação de materiais através da atribuição de metadados de conteúdo, como facilitará associação de materiais sobre o mesmo assunto, ampliando a estrutura de conhecimento descrita e explicitada.

\section{Considerações Finais}

O ensaio proposto neste artigo possibilitou sistematizar uma pequena parte dos novos conceitos geométricos adotados na arquitetura contemporânea, indicando a necessidade de detalhar e aprofundar a caracterização e definição dos conceitos identificados.
Uma melhor compreensão sobre alguns termos classificados se faz necessária, para que possam ser sistematizados processos de modelagem paramétrica das obras de arquitetura.

$\mathrm{Na}$ sequencia do trabalho irão ser estudadas e reconhecidas taxonomias mais específicas que abordem os conceitos matemáticos e as técnicas e tecnologias de modelagem paramétrica adequados para a definição geométrica de tais conceitos na arquitetura contemporânea.

\section{Agradecimentos}

Agradecemos ao CNPQ, órgão financiador dos projetos de pesquisa: O Processo de Ensino e Aprendizagem de Projeto mediado pelas Tecnologias da Informação e Comunicação em Arquitetura e Design/UFSC, que permitiu o desenvolvimento da rede TEAR_AD, e a qual este trabalho está associado; e PROBARQ - Produção e Compartilhamento de Objetos de Aprendizagem para 0 Projeto de Arquitetura/UFPEL, no qual foi desenvolvida a metodologia adotada neste trabalho. Agradecemos particularmente a Universidade Federal de Santa Catarina e ao PósArq (Programa de Pós-Graduação em Arquitetura e Urbanismo) pela oportunidade de realização do doutoramento em Arquitetura e Urbanismo.

\section{Referências}

Borda, A. Pires, J. F. Dalla Vecchia, L. Celani, G. (2010). Produção e Compartilhamento de Objetos de Aprendizagem Dirigidos ao Projeto de Arquitetura [Production and sharing of learning objects, aimed at architecture desing projects]. In: SIGRADI, Bogotá. Proceedings of the 14th Congress of the Iberoamerican Society of Digital Graphics. Bogotá: Ediciones Uniandes, v. 01. p. 104-107.

Burry.J. Burry, M.. (2010). The New Mathematics of Architecture. London: Thames e Hudson.

Chevallard, Y. (1999). El Análisis de las Prácticas Docentes en la Teoría Antropológica de Lo Didáctico. Recherches em Didactique de Mathématiques, Vol 19, no 2, pp. 221-266. (Traducción de Ricardo Barroso, Universidad de Sevilla). Retrieved from http://www.uaq.mx/matematicas/redm/art/a1005.pdf

Mitchell W. J. (1990). The Logic of Architecture. Cambridge: MIT Press. 
Monedero, J. Parametric design. A review and some experiences. Automation in Construction (2000). Retrieved from http://info.tuwien.ac.at/ecaade/proc/moneder/moneder.htm

Novo, H. F. (2007). A elaboração de taxonomia: princípios classificatórios para domínios interdisciplinares. Dissertação de Mestrado, Programa de Pós-graduação em Ciência da Informação, Universidade Federal Fluminense. 172 p.

Oliveira, L. C. Pereira, A. T. C. (2011). Mudanças metodológicas decorrentes da implantação recente de BIM em escritórios de arquitetura. In: XV SIGRADI - Congresso de la Sociedad Iberoamericana de Gráfica Digital, Santa Fé - Argentina. SIGRADI 2011 - Cultura Aumentada, v. XV. p. 134-137

Pires, J. F. Borda, A. S. (2009). Construção de Vocabulário e Repertório Geométrico para os estágios iniciais da prática Projetual de Arquitetura. In: XIX Simpósio Nacional de Geometria Descritiva e Desenho Técnico e VIII Conference on Graphics Engineering for Arts and Design - Linguagens e Estratégias da Expressão Gráfica: Comunicação E Conhecimento. Bauru: UNESP, v. 01. p. 1167-1181.

Pires, J. F. Construção do Vocabulário e Repertório Geométrico para o Projeto de Arquitetura. (2010). Dissertação de Mestrado, Programa de Pós-Graduação em Arquitetura e Urbanismo PROGRAU, UFPEL, Pelotas. 154 p. Retrieved from http://prograu.ufpel.edu.br/uploads/biblioteca/dissertacao_janice .pdf

Pires, J. F.; Borda, A. (2010). Identificação de estruturas de saber implícitas em materiais didáticos para a delimitação de uma taxonomia de domínio. In: $1^{\circ}$ Congresso Internacional de Educação a Distância da UFPel, Pelotas, Brasil.

Pottmann, H. Asperl, A. Hofer, M. Kilian, A. (2007). Architectural Geometry. Exton, Pensnsylvania: Bentley Institute Press.

Pottmann, H. (2010). Architectural Geometry as Design Knowledge. Archittectural Design, v. 80. p.72-77. Retrieved from http://onlinelibrary.wiley.com/doi/10.1002/ad.1109/abstract

Studer, R.; Benjamins, V. R.; Fensel, D. (1998). Knowledge Engineering: Principles and Methods. Data \& Knowledge Engineering, v. 25, n. 1-2, p. 161-197.

Vaz, C. V. (2011). Um método de ensino de projeto baseado no conhecimento: sistemas generativos e ontologias aplicadas no ensino de arquitetura paisagística. Tese de Doutorado, Programa de Pós-Graduação em Arquitetura e Urbanismo, Universidade Estadual de Campinas.

Woodbury, R. (2010). Elements of Parametric Design. London: Routledge. 